\title{
Mining biomarker information in biomedical literature
}

Erfan Younesi ${ }^{1,2}$, Luca Toldo ${ }^{3}$, Bernd Müller ${ }^{1}$, Christoph M Friedrich ${ }^{1,5}$, Natalia Novac ${ }^{3}$, Alexander Scheer ${ }^{4}$, Martin Hofmann-Apitius ${ }^{1,2}$ and Juliane Fluck ${ }^{1 *}$

\begin{abstract}
Background: For selection and evaluation of potential biomarkers, inclusion of already published information is of utmost importance. In spite of significant advancements in text- and data-mining techniques, the vast knowledge space of biomarkers in biomedical text has remained unexplored. Existing named entity recognition approaches are not sufficiently selective for the retrieval of biomarker information from the literature. The purpose of this study was to identify textual features that enhance the effectiveness of biomarker information retrieval for different indication areas and diverse end user perspectives.
\end{abstract}

Methods: A biomarker terminology was created and further organized into six concept classes. Performance of this terminology was optimized towards balanced selectivity and specificity. The information retrieval performance using the biomarker terminology was evaluated based on various combinations of the terminology's six classes. Further validation of these results was performed on two independent corpora representing two different neurodegenerative diseases.

Results: The current state of the biomarker terminology contains 119 entity classes supported by 1890 different synonyms. The result of information retrieval shows improved retrieval rate of informative abstracts, which is achieved by including clinical management terms and evidence of gene/protein alterations (e.g. gene/protein expression status or certain polymorphisms) in combination with disease and gene name recognition. When additional filtering through other classes (e.g. diagnostic or prognostic methods) is applied, the typical high number of unspecific search results is significantly reduced. The evaluation results suggest that this approach enables the automated identification of biomarker information in the literature. A demo version of the search engine SCAIView, including the biomarker retrieval, is made available to the public through http://www.scaiview.com/scaiviewacademia.html.

Conclusions: The approach presented in this paper demonstrates that using a dedicated biomarker terminology for automated analysis of the scientific literature maybe helpful as an aid to finding biomarker information in text. Successful extraction of candidate biomarkers information from published resources can be considered as the first step towards developing novel hypotheses. These hypotheses will be valuable for the early decision-making in the drug discovery and development process.

Keywords: Text-mining, Biomarker discovery, Information retrieval, Terminology

\footnotetext{
* Correspondence: juliane.fluck@scai.fraunhofer.de

'Department of Bioinformatics, Fraunhofer Institute for Algorithms and Scientific Computing (SCAI), Schloss Birlinghoven, Sankt Augustin 53754, Germany

Full list of author information is available at the end of the article
} 


\section{Background}

During the past years, high-throughput technologies have been extensively employed for the study of molecular mechanisms underlying different diseases; this has led to the discovery of a large number of molecular biomarkers [1]. The US National Institutes of Health defines a biomarker as "a characteristic that is objectively measured and evaluated as an indicator of normal biological processes, pathogenic processes or pharmacological responses to a therapeutic intervention" [2].

Depending on representing molecular, physiological, or structural features, biomarkers show significant diversity - spanning from genes, proteins, DNA, RNA, and SNPs to blood cholesterol levels and patterns of brain abnormality. Due to such diverse coverage, ambiguity in defining biomarkers types and classes continues to exist [3-5].

Application of biomarkers, however, goes beyond disease prediction and monitoring; in fact they have also been utilized throughout various stages of drug discovery and development [6]. For example, biomarkers play an important role in drug target discovery and validation (e.g. as quantitative readouts for candidate drugs) [7], in the monitoring of toxicity mechanisms (e.g. assessment of indication of unwanted side-effects) [8], and noninvasive imaging of diseased organs [9]. In the process of drug development, biomarkers are considered to be pivotal for informed go/no-go decision-making in the early stages of drug development [10]. For example, mechanistic biomarkers can be used to pre-clinically measure a drug's pharmacological activity in terms of its distribution and interaction with a defined protein target. Such measurements help to decide whether to move forward to the next phase of clinical development.

A first step to finding supportive evidence for clinically important potential biomarkers is to search the accumulated data and knowledge generated from basic research [11]. For efficient exploration of the suspected large amount of biomarker information contained in the biomedical literature, semantic search and information retrieval systems are of utmost importance. The largest publicly available biomedical literature resource, PubMed, makes use of MeSH (Medical Subject Headings) concepts to annotate the abstracts so that it maps these concepts to user queries and by this means allows for semantic search [12]. However, to search for reported biomarkers, (e.g. genes, proteins, or genetic variations) in a text corpus, additional annotation of these entities and their normalization to relevant databases is required. Such a search is currently not provided by PubMed. Manual annotation is also time consuming and lags behind due to the ever abundance of new publications. For example, compiling a compendium of potential biomarkers for pancreatic cancer was carried out by systematic manual curation of the literature and took over 7,000 person hours [13].

The automated identification of relevant terminology, a process known as Named Entity Recognition (NER), can support semantic annotation and information retrieval. In the past years, several NER methods for the extraction of different biological entities have been developed, primarily focusing on the recognition of gene and protein names. In this regard, the BioCreAtIvE assessments present an overview about the state of various technologies and approaches in use [14-16]. NER approaches have already been used to support the identification of biomarker genes. In [17], the authors applied gene and protein name recognition techniques to Medline and OMIM (Online Mendelian Inheritance in Man) to identify potential serum biomarkers for Down syndrome. Similarly, a named entity tagging approach was employed to search for prostate cancer biomarker candidate genes in OMIM records [18]. Studies on biomarker relation extraction from text have considered the extraction of semantic relations between diseases and genes or proteins $[19,20]$. In a recent study, an information extraction framework has been developed for the classification of biomarker sentences that showed favourable results, but the study was unfortunately focused on a small training set [21]. Moreover, biomarker information is often dispersed over the entire abstract, making it difficult to reach high information recall with sentence classification systems.

The aim of this work is to analyze how information about potential biomarkers is expressed in the complete body of a scientific text, and identify additional textual features that are relevant for the retrieval of potential biomarker information from the scientific literature.

\section{Methods}

\section{Information retrieval system and entity ranking}

Our retrieval system is composed of two software components: the named entity recognition tool ProMiner and the knowledge discovery framework SCAIView $[22,23]$. Figure 1 shows the design overview of the biomarker information retrieval system. ProMiner is one of the systems for gene normalization, which performed very well in BioCreAtIvE I and II assessments [24-26], reaching an F-score of 0.8 for the recognition and normalization of human genes and proteins. The ProMiner system has been designed for the semi-automated generation of dictionaries and the recognition of spelling variants, as well as the disambiguation of acronyms and common word synonyms.

The biomarker retrieval terminology and other dictionaries were incorporated into ProMiner, followed by ProMiner's annotation of all Medline abstracts using the 


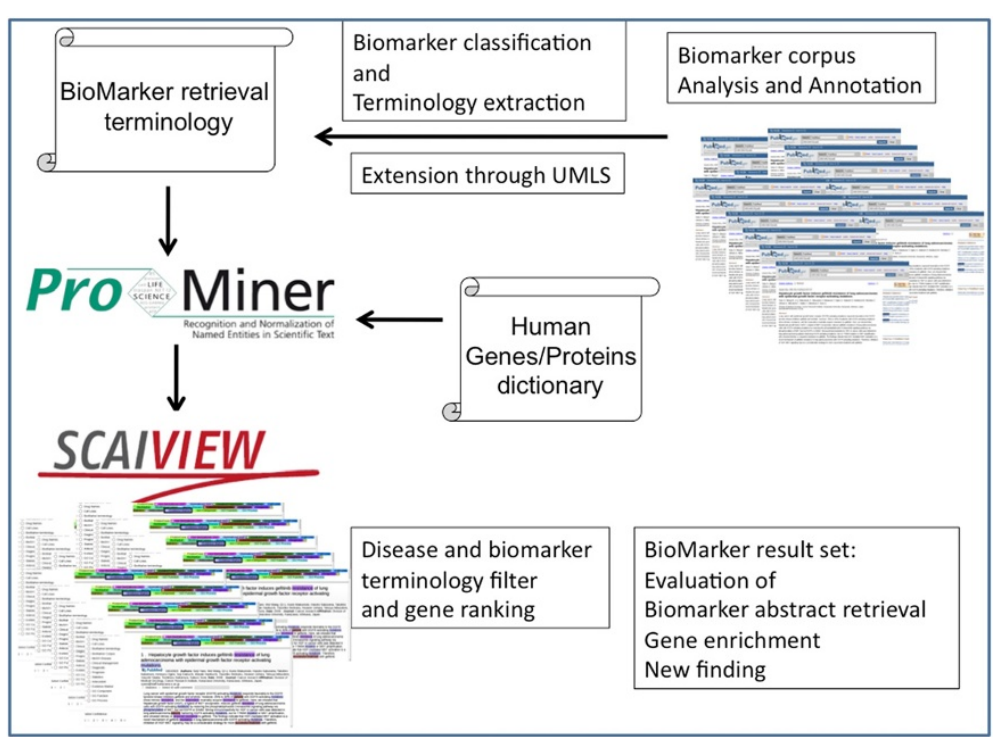

Figure 1 An overview of the biomarker retrieval system design.

\section{Overexpression of circulating $\overline{\mathrm{c} \text {-met }}$ messenger RNA is significantly correlated with nodal stage and early recurrence in non-small cell lung cancer.}

IM PubMed 16162743 Authors: Cheng, Tian-Lu; Chang, Mei-Yin; Huang, Sung-Yu; Sheu, Chau-Chyun; Kao, Eing-Long; Cheng, Yuven; Chong, Inn-Wen Date: 2005-09 Journal: Chest Affiliation: MedicoGenomic Research Center, Kaohsiung Medical University, 100 Shih-Chuan First Rd, Kaohsiung 807 Taiwan.

$\square$ Statistics $\square$ Select ID with comment:

BACKGROUND: The c-met receptor and its ligand hepatocyte growth factor have been shown to be involved in tumor invasiveness and metastasis Overexpression of $\overline{c-m e t}$ has been demonstrated in lung cancer tissues and cell lines, but the expression of $\bar{c}$-met in peripheral blood (circulating $\underline{c}$-met) has not been addressed. The molecular monitoring of circulating $\overline{c-m e t}$ could be helpful for selecting patients for adjuvant therapy.

OBJECTIVES: To investigate the expression of circulating $\overline{c-m e t}$ in hon-small cell lung cancer (NSCLC) patients and to assess its prognostic implications.

METHODS: We quantified the levels of c-met messenger RNA (mRNA) in paired tumor and normal lung tissues and their peripheral bloods in 45 patients with NSCLC by real-time polymerase chain reaction (PCR). The expression status of c-met protein in fumor tissues was further evaluated by immunohistochemistry.

RESULTS: C-Met mRNA was significantly higher by 1.5 to 11 times in 34 of 45 tumor tissues $(75.5 \%)$ than it was in their normal counterparts by real-time PCR. A comparison of this assay to immunohistochemistry suggested that real-time PCR was more sensitive than immunohistochemistry $(27$ of 45 tumor tissues, $60.0 \%)$ for the detection of $\bar{c}$-met $(p=0.016)$. Of these patients with overexpression of $\mathrm{c}$-met in tumors. $67.6 \%$ ( 23 of 34 patients) expressed higher amounts of circulating $\mathrm{c}$-met by 1.4 to 8 times that of the normal control subjects. In addition, overexpression of circulating $\mathrm{c}$-met was significantly correlated with nodal $(N)$ stage $(p=0.011)$, but weakly correlated with fumor $(T)$ stage $(p=0.056)$ and overall stages $(p=0.054)$ in patients with NSCLC. However, no correlations were found among circulating $\overline{c-m e t}$ and other factors such as age, gender, and pathologic types. Moreover, by univariate analysis, circulating $\mathrm{c}$-met overexpression and pathologic stages (including $\mathrm{T}$ and $\mathrm{N}$ stages) were the most important factors correlated with early recurrence $(p<0.05)$. Only the circulating $\mathrm{c}$-met remained as an independent predictor of early recurrence (hazard ratio. $3.94 ; 95 \%$ confidence interval. 1.17 to 13.33; $p=0.027$ ) after Cox regression multivariate analysis.

CONCLUSIONS: Overexpression of circulating $\overline{c-m e t}$ is significantly correlated with the $\mathrm{N}$ stage and early recurrence. Moreover, early recurrence is frequently noted in patients with overexpression of circulating $\mathrm{c}$-met, indicating that circulating $\underline{c}$-met is an independent negative prognostic indicator in NSCLC.

Figure 2 Example annotation of a biomarker abstract in SCAIView. 
dictionaries. The resulting entity annotations are stored in an Apache Lucene(TM)-based search index, together with the documents and their meta-information. SCAIView incorporates the Lucene-based index and allows for searches that include exact matches, wildcard options, and Boolean operations. The document visualization within SCAIView provides highlighting for all the entity classes with tooltips containing available linkouts, descriptions, and depictions (Figure 2).

The annotations are organized in the form of hierarchies (semantic trees) and can be navigated by the user via selecting full classes (e.g. 'genes' or 'diseases'), selecting certain subclasses (e.g. 'Nervous Systems Diseases'), or singular dictionary entries (e.g. 'Alzheimer's Disease'). For Boolean operations, either single entity class or complete tree classes could be selected. For example, the query “'Alzheimer's Disease' AND 'Evidence marker' " would retrieve all abstracts that contain one or more terms of both terminology classes. By selecting the Human Genes/Proteins, an entity table containing a list of the recognized genes and proteins in the selected corpus would be displayed (Figure 3).

The biomedical entities are ranked either by frequency or by relative entropy (in descending order of importance). The frequency ranking orders the entities with respect to the number of citations. The relative entropy ranking is based on the Kullback-Leibler divergence (also known as information gain) [27,28], and calculates the fraction between the documents containing the entity in the result set and the total number of documents containing the entity in the complete Medline document set. The formula for the relative entropy is given as:

$$
R E\left(p_{1}, p_{2}\right)=p_{1} \cdot \log \frac{p_{1}}{p_{2}}=p_{1} \cdot\left(\log p_{1}-\log p_{2}\right)
$$

where $p_{1}$ is the number of abstracts containing the entity in the query selected corpus and $\mathrm{p}_{2}$ denotes the total number of documents in which the entity occurs within an unspecific reference corpus (i.e. the entire Medline). The Kullback-Leibler divergence ranks those entities high, which have especially high frequency in the selected corpus in comparison to the unspecific reference corpus. This means that frequently occurring entities do not receive high ranks. For example, using the query " Alzheimer's Disease' AND 'Evidence marker' AND 'Human Genes/Proteins' ", we retrieved 331 abstracts containing IL1B with a frequency ranking of 10. Conversely, according to the relative entropy formula, IL1B has an entropy rank of 34 despite its high occurrence in Medline (i.e. 40685 abstracts).

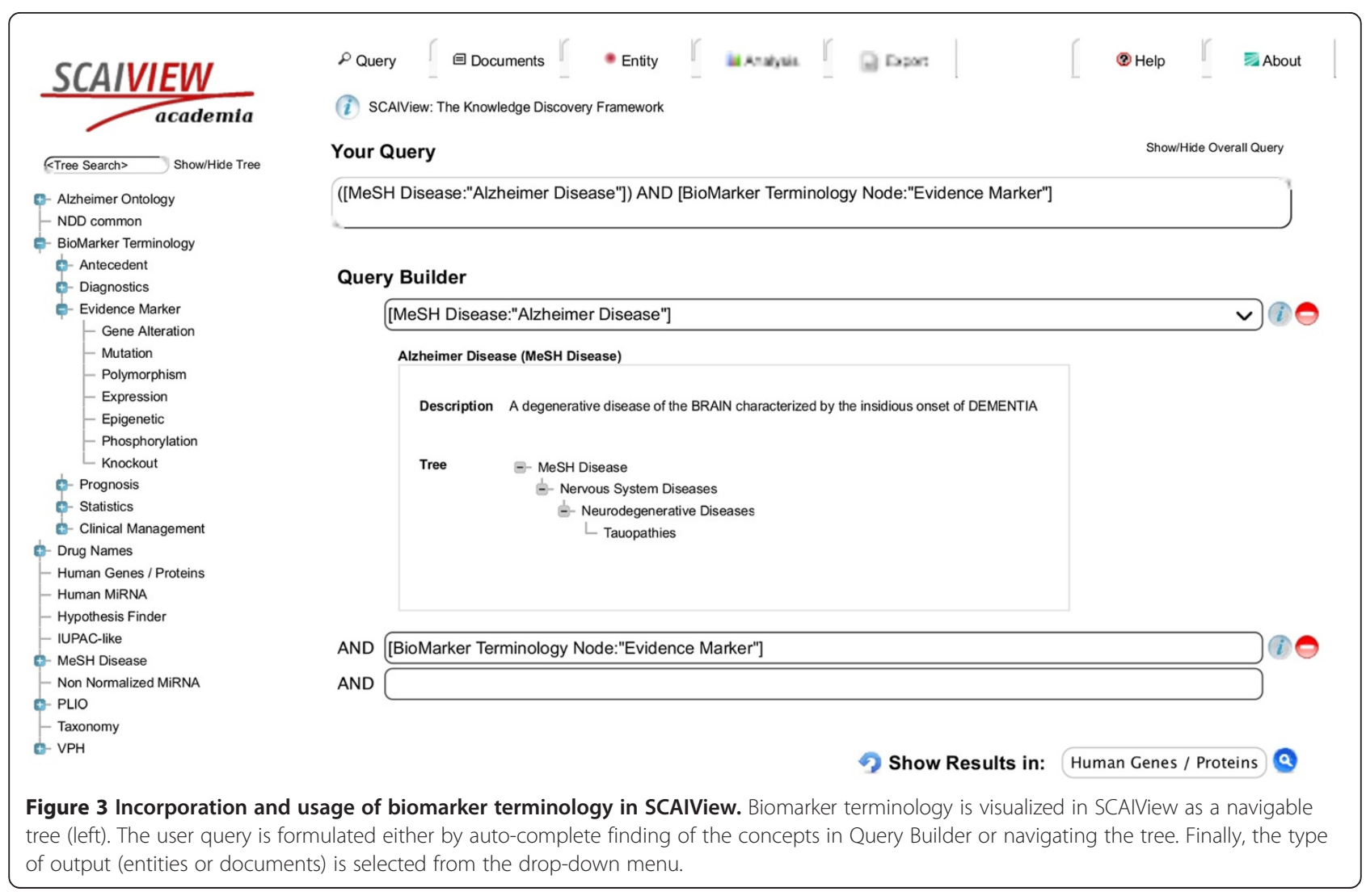




\section{Corpus annotation and terminology generation}

Five end users were asked to provide 50 relevant abstracts containing cancer or drug-related biomarker information. The abstracts were chosen from the following areas due to data availability and their current interest in treating cancer.

NSCLC (Non-Small Cell Lung Cancer) related, Breast cancer and predictive/prognostic, Met signaling and cancer, EGFR related, Gefitinib related, and Erbitux related.

A training corpus of 289 abstracts was established (see Additional file 1 for PMIDs of the training abstracts), and two annotators (different from the end users) were asked to annotate 10 abstracts out of this corpus in a first step. The annotators manually marked all biomarker information evidence that were perceived to be relevant (see Figure 2 for an example of automatic annotations). Instead of annotating the biomarkers themselves (i.e. the gene, protein, or SNP mentions), the biomarker evidence was annotated and assigned to six classes. These classes were chosen based on the conventional classification of biomarker types as well as the distribution analysis of the potential biomarker information in the literature. Subsequently, the following simple annotation guideline was generated:

- Clinical Management: annotate all terms indicating clinical investigations in patients, which includes the initial mentioning, the clinical study, and finally the treatment

- Diagnostics: annotate all diagnostics that are used, which includes the initial disease stage, the molecular identification, and blood diagnostics

- Prognosis: annotate all terms indicating any prognosis, outcome, or marker (e.g. clinical or biomarkers, adverse effects, resistance, response, disease progression or outcome)

- Evidence marker: annotate all changes in gene and protein abundance, spanning from expression to mutation, SNP variations to phosphorylation status

- Antecedent: annotate all risk factors mentioned for the relevant disease

Using this guideline, both annotators annotated 50 abstracts independently, and resulting agreement between them was measured based on a kappa value. A kappa value measures the degree of confidence between the two annotators as follows [29]:

$$
\text { kappa }=\frac{(\operatorname{Pr}(\mathrm{a})-\operatorname{Pr}(\mathrm{e}))}{(1-\operatorname{Pr}(\mathrm{e}))}
$$

where $\operatorname{Pr}(\mathrm{a})$ is the relative observed agreement among the raters and $\operatorname{Pr}(\mathrm{e})$ is the probability of random agreement. The remaining abstracts were then annotated by one of the annotators.

\section{Performance evaluation}

For all evaluations, our search for Alzheimer's disease (AD) or multiple sclerosis (MS) scenarios was expanded using MeSH synonyms and matches of the human gene/ protein dictionary. The ranked gene list provided by SCAIView was compared with the gold standard gene set that we obtained for AD and MS from the BIOBASE database [30]. This database contains manually curated literature evidence for causal and correlative associations of genes and proteins to human diseases. Both gold standards were created by filtering property reports, based on the disease indication, and then importing the resulting list of genes and proteins to a text file based on their supporting evidence (i.e. experimentally determined). To evaluate the performance, a gene enrichment ranking methodology adopted from the Gene Set Enrichment Analysis (GSEA) was applied [31]. The goal of the enrichment is to determine whether genes within the gold standard (gene set $\mathrm{S}$ ) are randomly distributed throughout the ranked list of retrieved genes $\mathrm{L}$ (with length $\mathrm{N}$ ) or primarily found at the top or bottom of the list. Computed score is the maximum deviation from zero when walking down the list of genes $L$ that is increased by $X_{j}=1$ every time a gene in the list $L$ is in $S$. Thus, the enrichment score (ES) is calculated as:

$$
E S=\max _{1 \leq i \leq N} \sum_{j=1}^{i} X_{j}
$$

Knowing that the output of the retrieval system is a list of genes/proteins that are ranked according to their relative entropy scoring, different combinations of the biomarker terminology classes were applied. For all combinations, values of precision, recall and F-score were calculated. In order to compare the ranked gene list with the gold standard, the F-score criterion was used. An F-score is computed as the following:

$$
\mathrm{F}-\text { score }=\frac{2 \times \text { Precision } \times \text { Recall }}{(\text { Precision }+ \text { Recall })}
$$

where Precision is defined as $\mathrm{TP} /(\mathrm{TP}+\mathrm{FP})$ and Recall is defined as TP/(TP+FN) [32], and TP = True Positive, $\mathrm{FP}=$ False positive, $\mathrm{TN}=$ True Negative $(\mathrm{TN})$ and $\mathrm{FN}=$ False Negative (FN).

From the list of retrieved entities, eleven top ranking genes/proteins were selected for an analysis, assessing how much biomarker information content is contained in their corresponding abstracts. Since more than one abstract is retrieved per gene and protein, and because a typical user might base its decision on the first retrieved 
abstracts (the most recent publications), only the first ten abstracts were taken into account. Subsequently, and after manually checking of abstracts, the number of true positive and false positive biomarker abstracts was determined.

\section{Results}

\section{Development of biomarker retrieval terminology}

The training corpus containing cancer or drug-related biomarker information was pre-annotated using gene protein and disease named entity recognition. In the next step, we investigated if we could identify additional terminology relevant to biomarkers from the abstracts. Initially two annotators highlighted relevant parts in 10 abstracts and discussed the annotations. As a result, six terminology classes were defined representing the relevant content for biomarker identification (Table 1). Abstracts containing information about potential gene biomarkers most often comprise evidence of gene or protein alteration as well as indication words for the clinical management or investigation. Moreover, diagnostic methods and prognostic terms are found frequently in these abstracts. Statistic evaluations strengthen the relevance of biomarkers and in some cases antecedents are strong indicators for disease status or therapy decisions (see Figure 2). Based on those terminology classes and a simple annotation guideline, all 10 abstracts were again annotated and discrepancies were discussed until a common understanding was reached between annotators. In the next step, the two annotators annotated 50 abstracts and the interannotator agreement was calculated. The kappa value of 0.81 showed that both annotators were in good agreement when annotating concepts represented by the six classes. Inconsistencies were mainly because of missed annotations (e.g. the concept 'patient' was annotated 3 times instead of 4 times in one abstract), boundary differences (e.g. annotation of 'management of patients' versus annotation of 'patients') and some differences in the annotation of diagnostic terms (e.g. the concept 'Child's score' was only annotated by one annotator). Those differences were discussed and one annotator annotated the remaining abstracts.

All annotated terms in the training corpus were automatically extracted and integrated into a seed biomarker retrieval dictionary by manually assigning them to the six main classes. In the next step, the seed set was structured, forming a seed terminology containing 76 entity classes with 1132 different synonyms. After extension of the terminology with similar classes and synonyms from $\mathrm{MeSH}$ and UMLS as well as expert knowledge for diagnostic tests (after the analysis of larger disease- and biomarker-related text corpora), the biomarker retrieval terminology contained 119 entity classes with 1890 different synonyms.

Distribution analysis of different biomarker classes in relevant text corpora (annotations in abstracts) showed that every training abstract contains at least one biomarker retrieval term. Moreover, Clinical Management and Prognosis terms were found most frequently in the corpora, followed by Diagnostic, Evidence and Statistics terms (see Table 1, last column). The Antecedent class plays a minor role in the selected training data (recall of $20 \%$ ), which might be quite different for diseases in which antecedents play a role in biomarker search. The six terminology classes are integrated into the SCAIView Demo server and can be accessed through http://www. scaiview.com/scaiview-academia.html.

\section{Non-small cell lung carcinoma (NSCLC) as use case scenario}

As a base line for biomarker candidate retrieval, selection of the disease NSCLC in combination with the automated recognition of gene names in our retrieval system returns 10790 Medline abstracts mentioning at least one gene concept (2663 different gene entities in total). Without further specification, these numbers are far too high to inspect and the resulting abstracts and

Table 1 Biomarker retrieval terminology classes and coverage of the terminology in the annotated corpora

\begin{tabular}{|c|c|c|c|}
\hline $\begin{array}{l}\text { Terminology } \\
\text { class }\end{array}$ & Description & Examples & $\begin{array}{l}\text { Annotation found } \\
\text { in abstracts }\end{array}$ \\
\hline $\begin{array}{l}\text { Clinical } \\
\text { Management }\end{array}$ & Terms indicating clinical investigations on patients & Patient; Cohort study & $96 \%$ \\
\hline Diagnostics & Terms representing clinical as well as molecular diagnostics & $\begin{array}{l}\text { Immunohistochemistry; Emission } \\
\text { Tomography; Microarray }\end{array}$ & $85 \%$ \\
\hline Prognosis & $\begin{array}{l}\text { Terms indicating the prediction for a patient /kind of biomarker/ } \\
\text { outcome of therapies }\end{array}$ & $\begin{array}{l}\text { Surrogate end point; clinical response; } \\
\text { biomarker; predictor }\end{array}$ & $88 \%$ \\
\hline Statistics & $\begin{array}{l}\text { Statistical methods indicating the strength of the biomarker } \\
\text { relationship }\end{array}$ & $\begin{array}{l}\text { Chi(2) test; mean +/- SD; univariate } \\
\text { analysis; Kaplan-Meier Analysis }\end{array}$ & $48 \%$ \\
\hline Evidence & $\begin{array}{l}\text { Terms describing genetic/ molecular evidence for activity of a } \\
\text { gene }\end{array}$ & $\begin{array}{l}\text { Mutation; gene amplification; } \\
\text { polymorphism; expression }\end{array}$ & $82 \%$ \\
\hline Antecedent & Terms expressing exposure to hazardous agents and risk factors & Smoker; susceptibility; exposure & $20 \%$ \\
\hline
\end{tabular}


genes cover various aspects of NSCLC-gene relations not relevant for the search of NSCLC biomarker candidates. Putting additional restriction on the semantic search using the five biomarker retrieval classes (ignoring the class Antecedent) reduces the number of retrieved documents down to 1539, containing 877 genes. Further reduction to relevant genes is possible through the inclusion of frequency or relative entropy ranking thresholds. Ignoring all gene mentions occurring in less than 5 abstracts decreases the gene set to 126 genes. Spot checks of ten genes with low relative entropy values revealed that nine of these genes are true positive biomarker candidates (see Table 2).

One of the biomarker candidates is Mucin 1 for which 8 abstracts under the above- mentioned criteria were retrieved. Mucin 1 is involved in invasiveness, metastasis, and angiogenesis of NSCLC [33] and its expression and localisation in lung adenocarcinoma patients is altered compared to normal epithelial cells [34]. A further exemplified action using the biomarker retrieval terminology is the literature search for suitable Mucin-1 antibodies. This can be directly conducted by applying the biomarker retrieval terminology. Selection of the Diagnostic subclass Immunohistochemistry and the Evidence subclass Expression together with the NSLCL $\mathrm{MeSH}$ variants and the Mucin-1 gene concept retrieves 16 abstracts. In 11 abstracts immunostaining of Muc1 is directly stated but only 4 abstracts mention the specific antibodies (CA15-3, clone DF3 in PMID 17409826, HMFG2 in PMIDs 8980247, 2456176 and 1284790). A further analysis of the retrieved full text articles is necessary to retrieve in a next step all information about the antibodies utilized. Higher recall rates for different antibodies for this example could be reached with searches for a broader disease area like Lung Neoplasms (31 abstracts), the search with the Diagnostic subclass Immunohistochemistry in combination with the text search 'anti-Muc1'
(105 abstracts), or in combination with the gene concept Muc1 and the Evidence marker Expression (1055 abstracts).

These examples demonstrate the ability of the biomarker search engine - depending on the application area - to substantially increase recall or precision of the search results, maximizing the efficiency of public domain literature searches.

\section{Adaptation and evaluation of biomarker retrieval in neurodegenerative diseases area}

To demonstrate the applicability of the biomarker retrieval terminology in other disease indications independently of the oncology-focused training corpus, the performance of retrieval for biomarker-related abstracts from Medline was tested in the field of Alzheimer's disease and multiple sclerosis.

In a first test it became evident that the class Diagnostics shows a high diversity between cancer and neurodegenerative diseases and that this class has to be extended for the new disease areas. For instance, in the area of neurodegenerative diseases, we included the diagnostic imaging subtree of $\mathrm{MeSH}$ (after omitting microscopy and molecular imaging) and a number of very specific medical classification systems provided by experts (e.g. MS Functional Composite score). The final BioMarker terminology contains 164 classes and 2506 synonyms.

After this optimization for neurodegenerative diseases, in three evaluation steps we examined: i) to what extent biomarker class selection influences the biomarker gene enrichment in comparison to the content of independent gold standards (entity retrieval); ii) how many biomarker-enriched abstracts could be retrieved with the selection of our terminology (abstract retrieval); and iii) how far new biomarker genes/proteins information could be retrieved which are not provided by the gold standard (potential biomarker identification).

Table 2 Spot check of NSCLC genes/proteins for their relevance to biomarker applicability and evidence

\begin{tabular}{lllllll}
\hline $\begin{array}{l}\text { Gene or } \\
\text { protein }\end{array}$ & $\begin{array}{l}\text { No. of retrieved } \\
\text { document }\end{array}$ & $\begin{array}{l}\text { Relative entropy } \\
\text { rank }\end{array}$ & $\begin{array}{l}\text { Frequency based } \\
\text { rank }\end{array}$ & $\begin{array}{l}\text { Example } \\
\text { PMID }\end{array}$ & $\begin{array}{l}\text { Biomarker } \\
\text { applicability }\end{array}$ & $\begin{array}{l}\text { Biomarker } \\
\text { evidence }\end{array}$ \\
\hline P53 & 281 & 1 & 1 & 20521348 & Prognosis & Expression \\
VEGFA & 128 & 6 & 4 & 21964530 & Prognosis & Expression \\
BCL2 & 90 & 8 & 6 & 19560836 & Survival & Expression \\
PCNA & 47 & 11 & 10 & 21495034 & Prognosis & Expression \\
VEGFC & 22 & 16 & 21 & 19758816 & Recurrence & Expression \\
PTGS2 & 33 & 25 & 15 & 20592629 & Survival & Expression \\
CDKN1B & 12 & 50 & 52 & 15483027 & Prognosis & Expression \\
DNAJB4 & 2 & 100 & 344 & 16788156 & Survival & Expression \\
CDK2 & 5 & 342 & 112 & 10817513 & Prognosis & No impact \\
\hline
\end{tabular}

For the SCAIView search the MeSH disease "Carcinoma, Non-Small-Cell Lung" in combination with five biomarker retrieval classes (omitting the class Antecedent) were selected and the retrieved genes were ranked according the Relative Entropy or frequency. In spot tests the abstracts were analysed for containing biomarker information for the respective genes. 


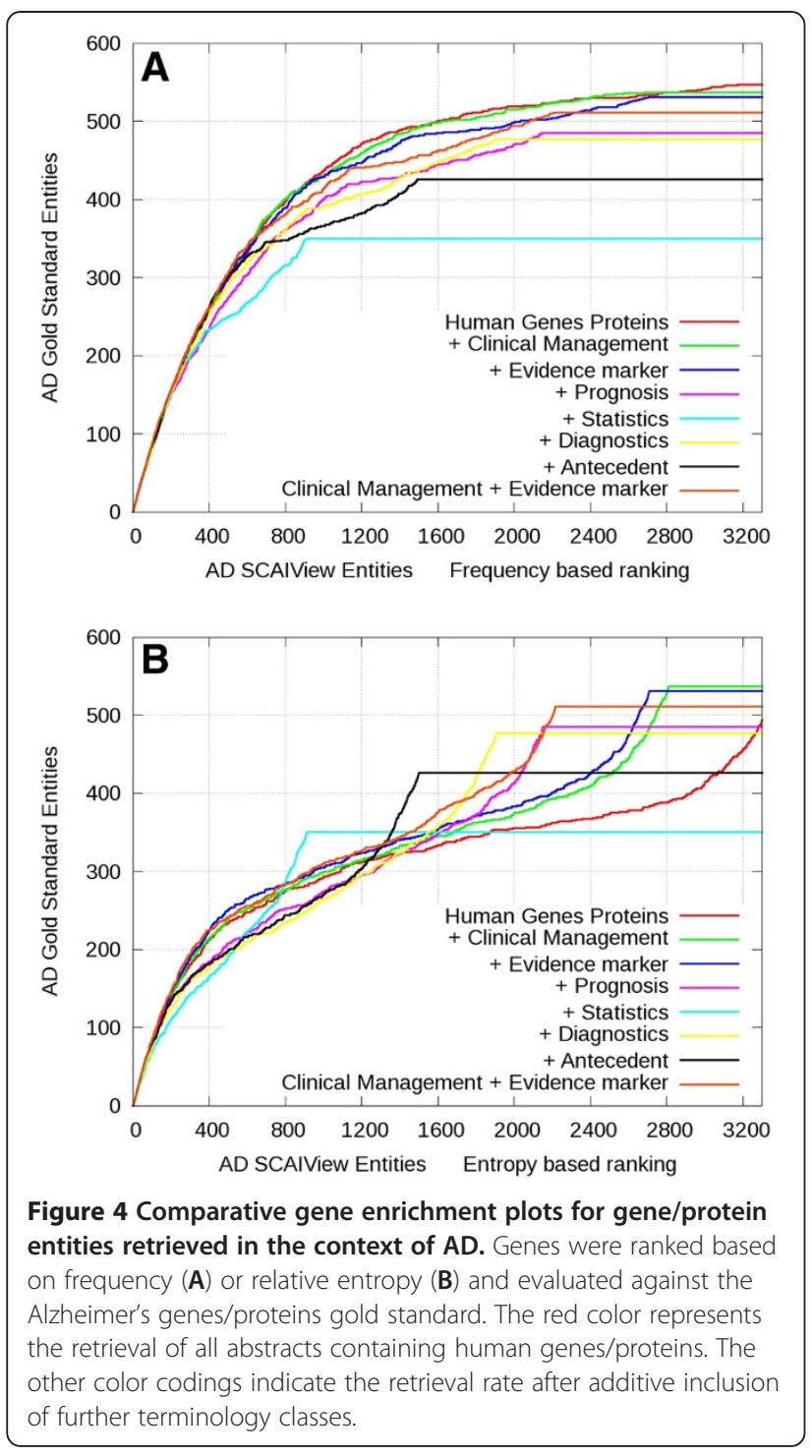

Using the gene enrichment ranking method and the selection of Human Genes/Proteins (our baseline approach), it turns out that almost all genes/proteins contained in the gold standard (95\% for Alzheimer's and 97\% for multiple sclerosis) were covered by the results of our retrieval system (see Human Genes/Proteins in Figures 4 and 5).

The system was able to successfully extract candidate biomarker genes/proteins relevant to the queried diseases and both ranking methods performed well for high-ranking genes. Nevertheless, the baseline search returned over 3600 genes from more than 33000 abstracts for Alzheimer's disease and over 2300 genes from more than 12900 abstracts for multiple sclerosis. This amount of abstracts for manual inspection is overwhelming. By additive selection of the Clinical Management or Evidence Marker classes, it can be shown that the relevant biomarker candidate genes are retrieved at
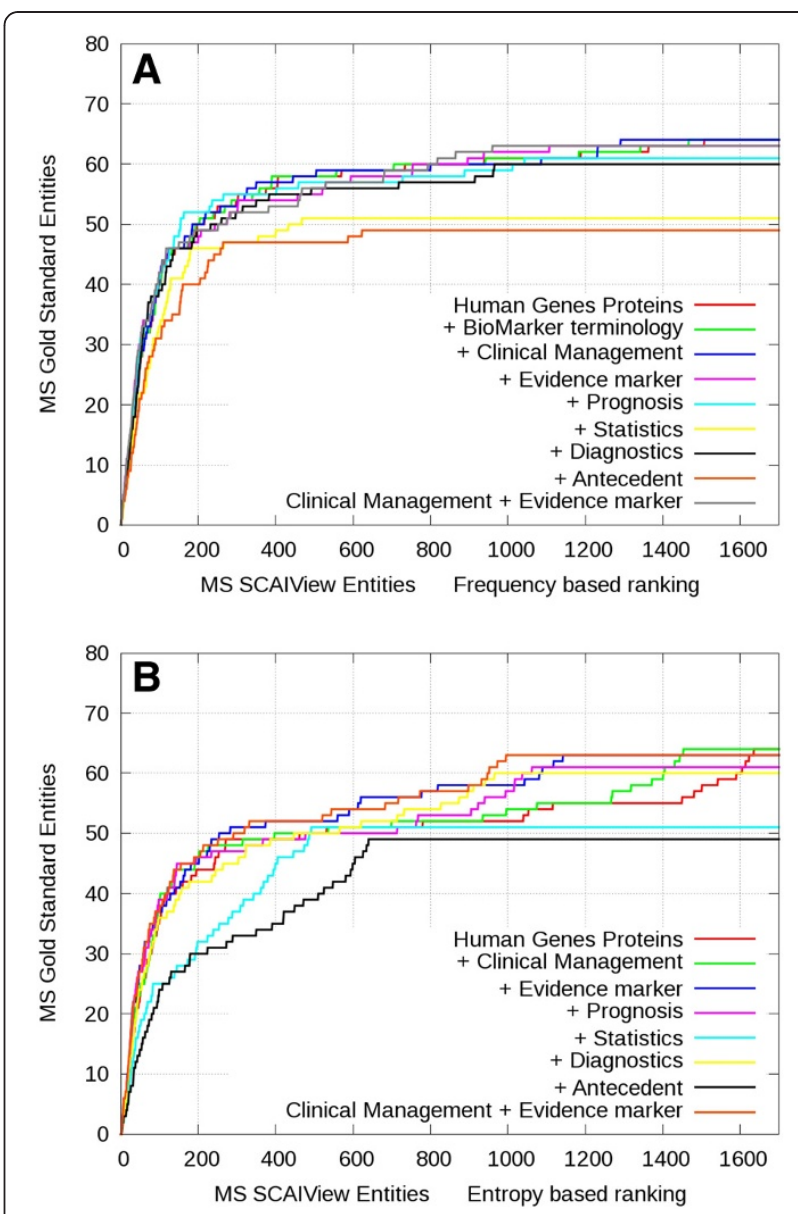

Figure 5 Comparative gene enrichment plots for gene/protein entities retrieved in the context of MS. Genes were ranked based on frequency (A) or relative entropy (B) and evaluated against the multiple sclerosis genes/proteins gold standard. The red color represents the retrieval of all abstracts containing human genes/ proteins. The other color codings indicate the retrieval rate after additive inclusion of further terminology classes.

higher ranks in both Alzheimer's and multiple sclerosis contexts (Figure 4 and Figure 5). For all other classes, the slope of gene enrichment decreases at lower ranks in comparison to selection of Human Genes/Proteins alone. Overall, frequency-based ranking (Figure 4A and Figure $5 \mathrm{~A}$ ) seems to perform better than relative entropy-based ranking (Figure 4B and Figure 5B). Although frequency-based ranking leads to higher gene enrichment slope between the ranks 300 and 1500, we observe a stronger enrichment at low ranking positions using relative entropy-based ranking. Analysis of those low ranking but frequent genes in Alzheimer's disease and multiple sclerosis shows that the high number of cytokines and inflammatory proteins are mere indicators of the disease. The frequency of those genes in the whole Medline is very high and for this reason they are underrepresented in the specific corpus. 


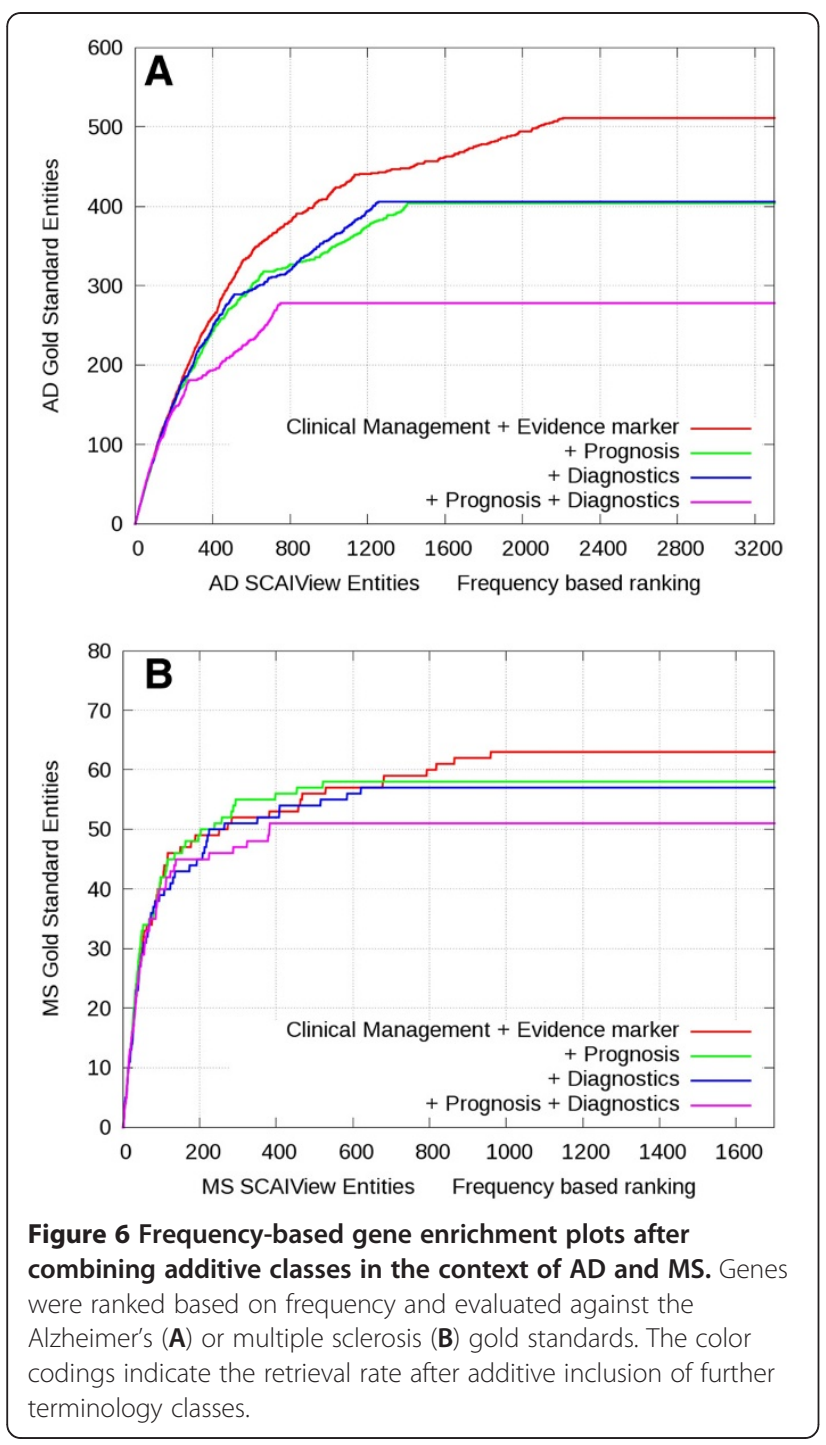

Using an AND combination of three or more terminology classes leads to similar enrichment slopes for higher ranks but with reduced recall (Figure 6). When compared to the MS gold standard, the combination of classes Clinical Management, Evidence Marker and Prognosis or Diagnosis performs even better than Clinical Management and Evidence Marker alone but loses some recall at lower ranks.

For Alzheimer's disease, we analyzed the outcome of the frequency based ranking method and calculated the precision, F-score and rank for different recall ranges (10\%, 30\%, and 50\% recall) and determined the maximal F-score (see Table 3). For the classes Clinical Management and Evidence Marker as well as for the combined selection of both classes, the values are similar but the maximal F-score (0.59 for Genes/Proteins alone and 0.58 for the combination) is reached at lower ranking positions (rank 555 for the combination instead of rank 728 for Genes/Proteins alone). For recall value of 0.3 , the precision is similar for all selections even with the combination of the classes Clinical Management, Evidence Marker and Prognosis. At recall value of 0.5, the precision drops down to 0.5 , otherwise 0.63 if the class Prognosis is not selected; moreover, the maximal F-score looses 7\% in comparison to Genes/Proteins alone.

The analysis of the retrieved abstracts for the first 10 ranked genes implies that the combination of different biomarker classes (Clinical Management and Evidence Marker alone or together with Diagnostics and Prognosis) leads to a reduction in the number of abstracts but to an increase in the number of remaining ones (Figure 7). At this point, the user has to decide about the trade-off between recall and precision. For genes with a high number of references (e.g. APP or APOE) a more stringent selection of abstracts is favorable, whereas for genes with a lower number of references a less restrictive search strategy might be beneficial.

\section{Analysis of novel candidates as potential biomarkers}

Genes retrieved by the selection of Clinical Management and Evidence Marker classes and not mentioned in the AD gold standard were checked manually by a biologist using the SCAIView environment. Such genes/proteins might be valuable for identification of novel biomarkers because they represent the yet-to-be identified biomarkers whereas those already matched with the gold standard are candidates that are better known as potential indicators of the disease. For this purpose, the abstracts must contain at least the information of the gene, which is altered (e.g. overexpression or mutation) in a particular state of $\mathrm{AD}$ and their therapeutic response state. Examples of such information with the corresponding PMIDs are given in Table 4.

For $\mathrm{AD}$, out of 400 genes, 158 genes/proteins had at least one evidence in the literature as being a potential biological indicator of Alzheimer's disease and thus were considered as true positives ( 40\%) whereas 241 genes/ proteins were recognized by the annotator as false positive genes $(\sim 60 \%)$ due to the lack of relevance to either Alzheimer's disease or biomarkers (Table 5).

\section{Discussion}

Protein biomarkers are required for informed decisionmaking in drug discovery and development. In order to evaluate and prioritize potential biomarkers, a systematic literature search is the first step. Abstracts containing information about potential gene biomarkers most often comprise evidence of gene or protein alteration as well as indication words for the clinical investigations. Moreover, diagnostic methods and prognostic terms are found frequently in these abstracts. Automated recognition of gene names, co-occurrence search with disease names 
Table 3 Performance evaluation for Alzheimer's disease

\begin{tabular}{|c|c|c|c|c|}
\hline Selection & Rank & Recall & Precision & F-score \\
\hline \multirow[t]{3}{*}{ Baseline: Genes / Proteins } & 60 & 0.10 & 0.92 & 0.17 \\
\hline & 230 & 0.30 & 0.73 & 0.42 \\
\hline & 469 & 0.50 & 0.61 & 0.55 \\
\hline Maximal F-score & 728 & 0.67 & 0.52 & 0.59 \\
\hline \multirow[t]{3}{*}{ Baseline + Clinical Management } & 61 & 0.10 & 0.90 & 0.17 \\
\hline & 226 & 0.30 & 0.75 & 0.42 \\
\hline & 465 & 0.50 & 0.61 & 0.55 \\
\hline Maximal F-score & 682 & 0.65 & 0.54 & 0.59 \\
\hline \multirow[t]{3}{*}{ Baseline + Evidence Marker } & 62 & 0.10 & 0.89 & 0.17 \\
\hline & 225 & 0.30 & 0.75 & 0.42 \\
\hline & 464 & 0.50 & 0.61 & 0.55 \\
\hline Maximal F-score & 654 & 0.62 & 0.54 & 0.57 \\
\hline \multirow[t]{3}{*}{ Baseline +Prognosis } & 63 & 0.10 & 0.87 & 0.17 \\
\hline & 247 & 0.30 & 0.68 & 0.41 \\
\hline & 541 & 0.50 & 0.52 & 0.51 \\
\hline Maximal F-score & 740 & 0.61 & 0.47 & 0.53 \\
\hline \multirow[t]{3}{*}{ Baseline + Diagnostics } & 64 & 0.10 & 0.86 & 0.17 \\
\hline & 237 & 0.30 & 0.71 & 0.42 \\
\hline & 494 & 0.50 & 0.57 & 0.53 \\
\hline Maximal F-score & 520 & 0.52 & 0.57 & 0.54 \\
\hline \multirow[t]{3}{*}{ Baseline + Statistics } & 64 & 0.10 & 0.86 & 0.17 \\
\hline & 227 & 0.30 & 0.74 & 0.42 \\
\hline & 678 & 0.50 & 0.42 & 0.45 \\
\hline Maximal F-score & 377 & 0.41 & 0.62 & 0.49 \\
\hline \multirow[t]{3}{*}{ Baseline + Clinical Management + Evidence Marker } & 60 & 0.10 & 0.92 & 0.17 \\
\hline & 224 & 0.30 & 0.75 & 0.42 \\
\hline & 451 & 0.50 & 0.63 & 0.56 \\
\hline Maximal F-score & 555 & 0.57 & 0.59 & 0.58 \\
\hline \multirow[t]{3}{*}{ Baseline + Clinical Management + Evidence Marker + Prognosis } & 61 & 0.10 & 0.90 & 0.17 \\
\hline & 230 & 0.30 & 0.73 & 0.42 \\
\hline & 568 & 0.50 & 0.50 & 0.50 \\
\hline Maximal F-score & 479 & 0.47 & 0.56 & 0.51 \\
\hline
\end{tabular}

Genes were ranked based on frequency and evaluated against the Alzheimer's gold standard. For the different selections recall, precision, F-score and rank has been estimated for 10,30 , and $50 \%$ recall. In addition the maximal recall has been estimated.

and their statistical ranking serve as a baseline for biomarker search.

Using our baseline approach, it turns out that almost all genes/proteins contained in the gold standard were covered by the results of our retrieval system. The system was able to successfully extract biomarker genes/ proteins relevant to the queried diseases and both ranking methods performed well for high-ranking genes. Nevertheless, the baseline search returned over 3600 genes from over 33000 abstracts for Alzheimer's disease and over 2300 genes from more than 12900 abstracts for multiple sclerosis. This amount of abstracts for manual inspection is overwhelming. Making use of the biomarker retrieval terminology developed here results in the significant reduction of the number of extracted genes and documents with almost no loss in the gene enrichment, especially for high-ranking genes. This indicates that inclusion of more biomarker classes in the search query helps to narrow down the retrieval results by adding more restrictive context to the search.

Concerning the statistical ranking of the result set, the frequency-based ranking method performs better than the relative entropy-based ranking for biomarker gene enrichment of the diseases investigated in this study. 


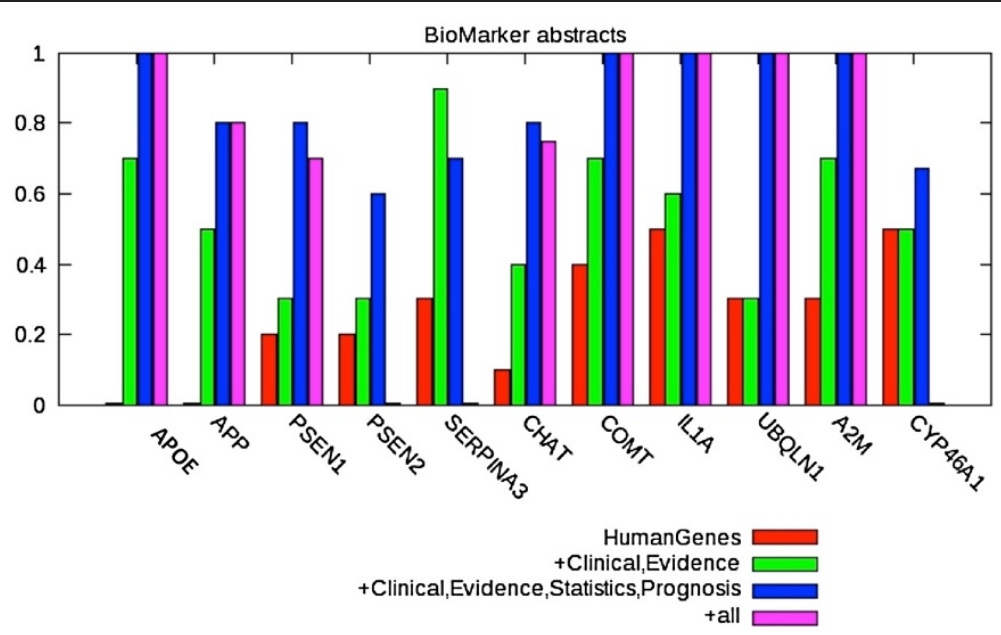

Figure 7 Proportion of retrieved abstracts containing biomarker information for Alzheimer's disease. The red color represents the retrieval of all abstracts containing human genes/proteins. The other color codings indicate the retrieval rate after additive inclusion of further terminology classes.

One explanation is that the relative entropy penalizes genes/proteins common to many investigations/publications and these entities rank at the end of the relevancy list, although they show a high frequency for the disease in question. Another explanation might lay in the selective annotation of entities by annotators of the external $\mathrm{AD}$ and MS gold standards; if annotators have selected the most frequent genes for annotation, the gold standard has a bias towards frequency ranking.

Evaluation of retrieved genes not existing in the gold standard for $\mathrm{AD}$ showed that almost half of these genes have probably the potential of being considered as potential biomarkers. This indicates that automated text mining using the biomarker terminology combinations increases recall for biomarker information retrieval and makes it possible to explore the biomarker space efficiently.

Comparing the number of retrieved entities between $\mathrm{AD}$ and MS shows that the biomarker information for each specific disease is distributed differently over the literature corpora. Thus, to obtain the optimum results, the search strategy should be adapted to each disease of interest; however, combining more general queries with the Statistics and/or Prognosis classes results in more stringent outputs in terms of biomarker specificity, sensitivity, and predictive parameters. For the purpose of new biomarker identification, it is more suitable to use less stringent combinations, which results in higher recall. In this case, the relative entropy-based ranking method can increase the likelihood of finding proper evidence in the support of a novel biomarker. For new disease areas, it is necessary to update the terminology especially for the class Diagnostics which shows the highest diversity of terminology in different disease areas. One possibility to improve the generalisation of the terminology would be to include all diagnostic classes from publicly available terminology resources.

Table 4 Examples of articles accepted to contain biomarker information

\begin{tabular}{|c|c|c|c|}
\hline PMID & Gene & Alteration & Textual evidence \\
\hline 17387528 & $\begin{array}{l}\text { ACAD8 } \\
\text { HMGCS2 }\end{array}$ & SNP & $\begin{array}{l}\text { In a European screening sample of } 115 \text { sporadic AD patients and } 191 \text { healthy control subjects, we } \\
\text { analyzed single nucleotide polymorphisms in } 28 \text { cholesterol-related genes for association with AD. } \\
\text { The genes HMGCS2, FDPS, RAFTLIN, ACAD8, NPC2, and ABCG1 were associated with AD at a } \\
\text { significance level of } P<\text { or }=0.05 \text { in this sample. }\end{array}$ \\
\hline 17531353 & SLC17A7 & $\begin{array}{l}\text { Protein expression } \\
\text { decrease }\end{array}$ & $\begin{array}{l}\text { Loss of VGLUT1 and VGLUT2 in the prefrontal cortex is correlated with cognitive decline in } \\
\text { Alzheimer disease. . . We quantified VGLUT1 and VGLUT2 in the prefrontal dorsolateral cortex } \\
\text { (Brodmann area 9) of controls and AD patients using specific antiserums. A dramatic decrease in } \\
\text { VGLUT1 and VGLUT2 was observed in AD using Western blot }\end{array}$ \\
\hline 19863188 & $\begin{array}{l}\text { HPX } \\
\text { SERPINF1 }\end{array}$ & $\begin{array}{l}\text { Cerebrospinal fluid } \\
\text { concentration }\end{array}$ & $\begin{array}{l}\text { Five differentially-expressed proteins with potential roles in amyloid-beta metabolism and vascular } \\
\text { and brain physiology [apolipoprotein A-1 (Apo A-1), cathepsin D (CatD), hemopexin (HPX), } \\
\text { transthyretin (TTR), and two pigment epithelium-derived factor (PEDF) isoforms] were identified. Apo } \\
\text { A-1, CatD and TTR were significantly reduced in the AD pool sample, while HPX and the PEDF } \\
\text { isoforms were increased in AD CSF }\end{array}$ \\
\hline
\end{tabular}


Table 5 Evaluation of genes not found in AD gold standard but retrieved using the biomarker terminology

\begin{tabular}{lll}
\hline $\begin{array}{l}\text { No. of genes/ proteins retrieved by SCAIView } \\
\text { but not contained in gold standard }\end{array}$ & $\begin{array}{l}\text { No. of genes with at least one } \\
\text { biomarker evidence in Medline }\end{array}$ & $\begin{array}{l}\text { No. of abstracts with lack of relevance either to } \\
\text { the disease or to being a biomarker }\end{array}$ \\
\hline 400 & 158 & 241
\end{tabular}

However, this might lead to a decline in retrieval precision. Additionally, some medical diagnosis scores are very specific and often do not occur in the large publicly available resources such as $\mathrm{MeSH}$ and UMLS.

The flexibility of the biomarker terminology in relation to different query formulations and different biomarker classes should be tested in future investigations.

\section{Conclusions}

The work presented in this paper is a first step towards developing a search engine for literature-based retrieval and in-silico validation of biomarker candidates. It was shown that the development and application of a dedicated biomarker terminology could enhance the retrieval performance significantly through combined search for genes and selected classes of the biomarker retrieval terminology. The experimental results, obtained in the course of this study, demonstrate the effectiveness of the proposed approach that provides a foundation in semantic indexing and retrieval.

\section{Additional file}

Additional file 1: List of the PMIDs of the abstracts used for evaluation of the system.

\section{Abbreviations \\ MeSH: Medical Subject Headings; NER: Names Entity Recognition; OMIM: Online Mendelian Inheritance in Man; UMLS: Unified Medical Language System; NSCLC: Non-Small-Cell Lung Carcinoma; AD: Alzheimer's Disease; MS: Multiple Sclerosis; PMID: PubMed Identifier.}

\section{Competing interests}

The authors declare that they have no competing interests.

\section{Authors' contributions}

EY carried out text annotations, participated in the terminology design, contributed to evaluations and drafted the manuscript. LT contributed to the study conception and design as well as data acquisition and analysis. BM carried out statistical analyses and maintained the softwares. CMF revised the results critically. NN participated in the design of the study, coordinated the expert annotations, and provided the use case scenario. AS conceived of the study. MHA participated in the design of the study, verified the terminology and revised the paper critically. JF participated in the design of the study and analysis of the results, coordinated the study, participated in evaluations and helped to draft the manuscript. All authors read and approved the final manuscript.

\section{Acknowledgements}

We wish to thank Caroline Kant from Merck Serono for her support and discussion in the generation of the terminology and Merck Serono experts for delivering the annotations. We would like to acknowledge Heinz Theodor Mevissen from Fraunhofer SCAI for ProMiner support, BIOBASE KL for disclosure of details in the article, and Dr. Karl Kirschner for proofreading the manuscript.

\section{Author details}

'Department of Bioinformatics, Fraunhofer Institute for Algorithms and Scientific Computing (SCAl), Schloss Birlinghoven, Sankt Augustin 53754 Germany. ${ }^{2}$ Bonn-Aachen International Center for Information Technology (B-IT), University of Bonn, Bonn, Germany. ${ }^{3}$ Knowledge Management, Operational Excellence \& Site Coordination, Merck Serono, Merck KGaA, Darmstadt, Germany. ${ }^{4}$ Informatics \& Knowledge Management, Merck Serono, Merck KGaA, Geneva, Switzerland. ${ }^{5}$ Department of Computer Science, University of Applied Science and Arts, Dortmund, Germany.

Received: 3 February 2012 Accepted: 10 December 2012 Published: 18 December 2012

\section{References}

1. Ghosh D, Poisson LM: Omics data and levels of evidence for biomarker discovery. Genomics 2009, 93:13-16.

2. Group BDW: Biomarkers and surrogate endpoints: preferred definitions and conceptual framework. Clin Pharmacol Ther 2001, 69:89-95.

3. Perera FP, Weinstein IB: Molecular epidemiology: recent advances and future directions. Carcinogenesis 2000, 21:517-524

4. Mayeux R: Biomarkers: potential uses and limitations. NeuroRx 2004, 1:182-188

5. Timbrell J: Types of biomarker and challenges for new biomarkers. Toxicol Lett 2006, 164(Suppl 1):S315.

6. Altar CA: The biomarkers consortium: on the critical path of drug discovery. Clin Pharmacol Ther 2008, 83:361-364.

7. Wagner JA: Strategic approach to fit-for-purpose biomarkers in drug development. Annu Rev Pharmacol Toxicol 2008, 48:631-651.

8. Marrer $\mathrm{E}$, Dieterle F: Impact of biomarker development on drug safety assessment. Toxicol Appl Pharmacol 2010, 243:167-179.

9. Bakhtiar R: Biomarkers in drug discovery and development. J Pharmacol Toxicol Methods 2008, 57:85-91.

10. Hurko H, Jones GK: Valuation of biomarkers. Nat Rev Drug Discov 2011 10:253-254.

11. Ongenaert $M$, Dehaspe L: Integrating automated literature searches and text mining in biomarker discovery. BMC Bioinforma 2010, 11(Suppl 5):05.

12. Krallinger $M$, Valencia $A$ : Text-mining and information-retrieval services for molecular biology. Genome Biol 2005, 6:224.

13. Harsha HC, Kandasamy K, Ranganathan P, Rani S, Ramabadran S, Gollapudi S, Balakrishnan L, Dwivedi SB, Telikicherla D, Selvan LDN, Goel R, Mathivanan S, Marimuthu R, DeCaprio JA, Srivastava S, Hanash SM, Htuban RH, Pandey A: A compendium of potential biomarkers of pancreatic cancer. PLOS Med 2009, 6:e1000046

14. BioCreAtlvE workshop. [http://www.biocreative.org].

15. Blaschke $C$, Hirschman $L$, Valencia A, Yeh A: A critical assessment of text mining methods in molecular biology. BMC Bioinforma 2004, 6(Suppl 1):S1-S23.

16. Hirschman L, Krallinger M, Wilbur J, Valencia A: The BioCreAtlvE II - critical assessment for information extraction in biology challenge. Genome Biol 2008, 9(Suppl 2):S1-S14.

17. Pennings $\mathrm{J}$, Koster MP, Rodenburg W, Schielen PC, de Vries A: Discovery of novel serum biomarkers for prenatal down syndrome screening by integrative data mining. PLoS One 2009, 4:e8010.

18. Deng X, Geng H, Bastola DR, Ali HH: Link test-a statistical method for finding prostate cancer biomarkers. Comput Biol Chem 2006, 30:425-433.

19. Bundschus M, Dejori M, Stetter M, Tresp V, Kriegel HP: Extraction of semantic biomedical relations from text using conditional random fields. BMC Bioinforma 2008, 9:207.

20. Elkin PL, Tuttle MS, Trusko BE, Brown HB: BioProspecting: novel marker discovery obtained by mining the bibleome. BMC Bioinforma 2009, 10(Suppl 2):S9

21. Islam MT, Shaikh M, Nayak A, Ranganathan S: Biomarker Information Extraction Tool (BIET) development using natural language processing and machine learning. In Proceedings of the International Conference and Workshop on Emerging Trends in Technology: 26-27 February 2010. Edited by 
Mishra BK, Kekre HB, Thampi GT, Gharpure P, Mukherji A, Lohani RB. Mumbai: ICWET; 2010:121-126.

22. Friedrich CM, Dach H, Gattermayer T, Engelbrecht G, Benkner S, HofmannApitius M: @neuLink: a service-oriented application for biomedical knowledge discovery. In Proceedings of Health Grid 2008; 2-4 June 2008. Edited by Solomonides T, Silverstein JC, Saltz J, Legre Y, Kratz M, Foster I, Breton V, Beck JR. Chicago: IOS Press; 2008:165-172.

23. Benkner S, Arbona A, Berti G, Chiarini A, Dunlop R, Engelbrecht G, Frangi AF, Friedrich CM, Hanser S, Hasselmeyer P, Hose RD, lavindrasana J, Köhler M, lacono LL, Lonsdale G, Meyer R, Moore B, Rajasekaran H, Summers PE, Wöhrer A, Woods S: @neurlST: infrastructure for advanced disease management through integration of heterogeneous data, computing, and complex processing services. IEEE Trans Inf Technol Biomed 2010, 14:1365-1377

24. Hanisch D, Fluck J, Mevissen HT, Zimmer R: Playing biology's name game: identifying protein names in scientific text. Pac Symp Biocomput 2003, 8:403-14.

25. Hanisch D, Fundel K, Mevissen HT, Zimmer R, Fluck J: ProMiner: rule based protein and gene entity recognition. BMC Bioinforma 2005, 6(Suppl 1):S14.

26. Morgan AA, Lu Z, Wang X, Cohen AM, Fluck J, Ruch P, Divoli A, Fundel K, Leaman R, Hakenberg J, Sun C, Liu HH, Torres R, Krauthammer M, Lau WW, Liu H, Hsu CN, Schuemie M, Cohen KB, Hirschman L: Overview of BioCreAtlvE II gene normalization. Genome Biol 2008, 9:S3.

27. Kullback S, Leibler R: On information and sufficiency. Ann Math Stat 1951 22:79-86

28. Büttcher $\mathrm{S}$, Clarke CLA, Cormack GV: Information retrieval: implementing and evaluating search engines. Cambridge, Mass. MIT Press, 296-298.

29. Smeeton NC: Early history of the kappa statistic. Biometrics 1985, 41:795.

30. BIOBASE BKL Proteome database. [http://www.biobaseinternational.com/ index.php?id=proteomedatabases].

31. Subramanian A, Tamayo P, Mootha VK, Mukherjee S, Ebert BL, Gillette MA, Paulovich A, Pomeroy SL, Gloub TR, Lander ES, Mesirov JP: Gene set enrichment analysis: a knowledge-based approach for interpreting genome-wide expression profiles. Proc Natl Acad Sci 2005, 102:15545-15550

32. Goutte C, Gaussier E: A probabilistic interpretation of precision, recall and F-score, with implication for evaluation. Advances in Information Retrieval. Lecture Notes in Computer Science 2005, 3408:345-59.

33. Szabo E: MUC1 expression in lung cancer. In Lung Cancer, Methods in Molecular Medicine. 3rd edition. Edited by Driscoll B. New Jersey: Humana Press; 2003:251-258. Volume 74.

34. Petty RD, Nicolson MC, Kerr KM, Collie-Duguid E, Murray Gl: Gene expression profiling in non-small cell lung cancer, from molecular mechanisms to clinical application. Clin Cancer Res 2004, 10:3237.

doi:10.1186/1472-6947-12-148

Cite this article as: Younesi et al: Mining biomarker information in biomedical literature. BMC Medical Informatics and Decision Making 2012 12:148.

\section{Submit your next manuscript to BioMed Central and take full advantage of:}

- Convenient online submission

- Thorough peer review

- No space constraints or color figure charges

- Immediate publication on acceptance

- Inclusion in PubMed, CAS, Scopus and Google Scholar

- Research which is freely available for redistribution 